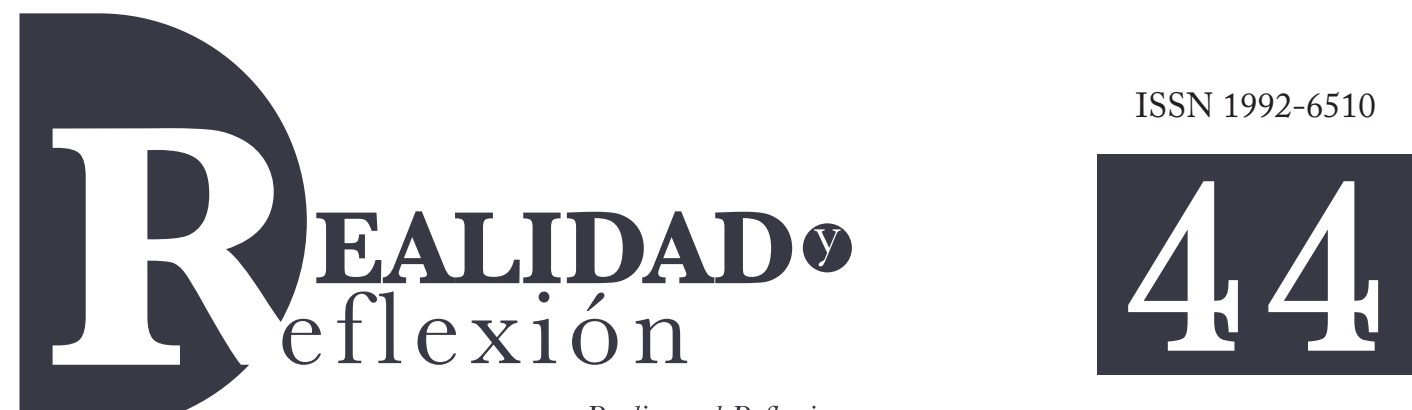

Reality and Reflection

Año 16, N 44, San Salvador, El Salvador, Centroamérica. Revista Semestral Julio-Diciembre 2016

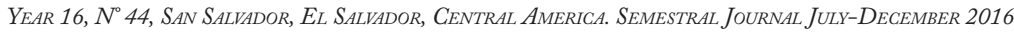

\title{
La gamificación como estrategia metodológica en el contexto educativo universitario
}

\author{
The gamification as a methodological strategy in the \\ university educational context
}

\author{
Herberth Alexander Oliva \\ Bachelor of Education. Southern Texas University. \\ Maestro en Administración de la Educación, Universidad Pedagógica de El Salvador. \\ Posgrado en Pedagogía de la Violencia Escolar. Tel Aviv University. \\ Posgrado en Educación para el Siglo XXI, Universidad del Valle Guatemala \\ Investigador para el área educativa, ICTI-UFG \\ holiva@ufg.edu.sv
}

\section{RESUMEN}

La gamificación como estrategia metodológica en el contexto educativo universitario es un artículo de naturaleza educativa que busca una aproximación interpretativa sobre la forma de ver, entender y aplicar la gamificación al proceso de enseñanza en el aula universitaria, buscando justificar el aporte académico de esta innovadora forma de adoptar los elementos del juego y utilizarlos con propósitos formativos.

Palabras clave: gamificación, educación universitaria, didáctica, estudiantes, aprendizaje, juegos.

\section{ABSTRACT}

The gamification as a methodological strategy in the university educational context, it is an article of educational nature that seeks an interpretative approximation on how to see, understand and apply gamification to the teaching in the university classroom, seeking to justify the academic contribution of this innovative how to adopt elements of the game and use them for training purposes.

Keywords: gamification, university education, didactics, students, learning, games. 


\section{A modo de introducción}

\section{La gamificación como estrategia metodológica}

Desde el análisis académico que debe abordarse en el tema de la calidad metodológica del docente universitario contemporáneo, las universidades en el continente americano han tenido que valorizar seriamente un cambio de dirección en lo referente a la estructura didáctica del componente formativo docente; por tanto, es imperante recordar que es la institución universitaria la que se incorpora en procesos de formación profesional académica, en donde la educación es un factor decisivo en el bienestar de las personas.

Una educación de calidad hacia el interior del aula universitaria pasa por convertir el proceso de enseñanza y aprendizaje en un valor social y de calidad, en el que los programas educativos sean desarrollados por el docente de conformidad con la pertinencia de los conocimientos que se construyen y las competencias que se desarrollan, así como de la eficacia y eficiencia de las metodología centradas en el campo andrológico. Ante ello surge una nueva tendencia en el campo educativo el cual es necesario llevar hacia el interior del aula universitaria: nos estamos refiriendo a la metodología denominada gamificación. Al hablar de gamificación estamos frente a una idea central extraída del acervo académico del especialista en temas informáticos: el británico Nick Pelling, quien en el año 2002 inicia en el desarrollo de su trabajo, en el mundo de las computadoras, una vinculación de elementos propios del videojuego en el mundo de la programación informática. Sobre la idea de estructurar el conocimiento, en una taxonomía fielmente estructurada al juego Pelling manifestaba lo siguiente: "Creo firmemente que en la medida que eliminemos los criterios aburridos y netamente metodológicos de la enseñanza, podremos construir metodologías simplistas que conduzcan a aprendizajes de gran valor". ${ }^{1}$

De conformidad con la teoría académica que sostiene la base epistemológica de la gamificación como estrategia de innovación docente en el aula universitaria, una clase gamificada debe contar con el ideal de desarrollar el potencial humano de la calidad educativa, para lo que se busca en la dinámica del juego: un aliciente directo que despierte en el estudiantado el interés por aprender; la gamificación, por lo tanto, va buscando la apertura didáctica de establecer un sistema de enseñanza docente que promueva la formación, selección, actualización y evaluación del contenido curricular que se desarrolla. E1 objetivo general de gamificación como estrategia metodológica de la mejora docente en el aula universitaria, persigue incidir en forma positiva a que el estudiantado pueda lograr el cumplimiento a cabalidad de objetivos específicos de aprendizaje, por lo cual el docente debe incentivar a los educandos a aprender por medios gamificados, en los cuales se implemente una eficaz vinculación de los elementos del juego con la acción educativa.

Como cierre de este apartado, se presenta la opinión de Carlos Scolari, quien hace referencia puntual sobre cómo la gamificación va ganando terreno en el plano de la educación superior. Al respecto dice

1 NOTA del autor: Información extraída de Nick Pelling / abril 2014 (fragmento de entrevista) New York Times Newsletter. 
lo siguiente: "la comunidad educativa está viendo su mirada en la vinculación de la tecnología con la educación y ha empezado a valorar el potencial de la gamificación como un elemento auxiliar de la renovación metodológica del profesorado del siglo XXI". ${ }^{2}$ Ante el razonamiento anterior, es evidente que la gamificación, bien enfocada en el contexto aprendizaje, puede renovar el compromiso del estudiante por obtener altos rendimientos académicos siempre y cuando se apliquen mecánicas y dinámicas de juego cuyo potencial sea el de volver más dinámica la interacción entre el docente y el estudiante.

\section{Profundizando la claridad del concepto gamificación}

Sobre la base del reconocimiento académico de la gamificación como un componente redinamizador de la enseñanza universitaria, encontramos una serie de interpretaciones sobre lo que es gamificar y su bagaje enriquecedor a la teoría academia pedagógica. Para Roberth Staycy Linker Phells de Harvard Graduate School of Education, gamificación es "recurrir al uso de elementos que forman parte de la estructura del juego, para lo cual aplican técnicas de juegos en contextos educativos, sigo creyendo fielmente que gamificar es aplicar esta estrategia metodológica en una herramienta de apoyo docente que logre despertar motivación en el estudiantado con el fin que sus procesos de aprendizaje sean significativos y exitosos". 3

2 SCOLARI Carlos A.; Homo Videoludens 2.0. De Pacman a la gamificacion, Col-lecció Transmedia XXI, Universitat de Barcelona, Barcelona Ed. 2013.

3 Información extraída de LINKER PHELLS, Roberth Staycy Linker; The Harvard Graduate School of Education /2015 Conference Cambridge.
Para la académica McGonigal Jane, la gamificación es "toda acción educativa en la cual el docente debe recurrir a la utilización de dinámicas, estructuras y mecánicas de juego en entornos y aplicaciones que no son precisamente un juego, buscando potenciar la motivación, la concentración, el esfuerzo, la fidelización y otros valores positivos comunes al modo y la forma en como aprenden los estudiantes". ${ }^{4}$

Una última interpretación del término gamificación la hace José Armando Mocis Valester, quien de manera puntual, argumenta que "la gamificación aplicada al entorno universitario, busca crear una dinámica del juego enfocada en la retroalimentación positiva del aprendizaje, en donde los estudiantes parten de la estructura jerárquica de clase en donde al cumplir objetivos y metas pueden acumular puntos, insignias y cualquier otra evidencia que denote el progreso significativo que tienen durante una clase". ${ }^{5}$

Al realizar un marco comparativo entre los autores mencionados, identificamos como Roberth Staycy habla de aplicar técnicas de juegos en contextos educativos; pero, por el otro lado, se observa cómo McGonigal hace hincapié en potenciar la motivación, la concentración, el esfuerzo y la fidelización para optimizar el modo como aprenden los estudiantes; sin embargo, la opinión de Mocis Valester viene a fortalecer las opiniones anteriores al argumentar que la gamificación aplicada al entorno universitario,

4 McGONIGAL, Jane; Reality is Broken. Why Games Make Us Better and How They Can Change the World, USA 2011.

5 MOCIS VALESTER, José Armando; GAMIFICACION: El último recurso de la actualización docente; Edit. Freymundh, Universidad Mayor de San Andrés, La Paz Bolivia 2015. 
busca crear una dinámica del juego, enfocada en la retroalimentación positiva del aprendizaje. Más allá de la visión interpretativa de estos autores, es sumamente gratificante el hecho de que todos coinciden en la idea que la gamificación recurre a la utilización de estrategias del juego, para incentivar un aprendizaje sobre el criterio de enseñar bajo situaciones de estudio, con lo cual se canaliza una mejor comunicación entre docente y estudiante, así como una ambientación del hecho educativo en el que enseñar y aprender se convierte en una acción más divertida y entretenida.

Como conclusión a este apartado, impera la noción académica que la gamificación, palabra españolizada que proviene del inglés gamification, es una dinámica que aglomera componentes que, en forma recurrente, vemos en juegos de video, en los que se pretende como fin último cómo la integración de una serie de dinámicas que permiten aumentar la participación de los estudiantes en sesiones educativas motivantes y útilmente entretenidas, hasta conseguir desarrollar un proceso formativo que pueda llenar las expectativas del "querer aprender" por parte del sector estudiantil.

\section{La gamificación como pilar de eficiencia y eficacia en el rendimiento educativo universitario}

Sustentar la introducción de una estrategia metodológica docente ante una diversidad de estilos de aprendizaje por parte de los estudiantes no es tarea fácil; no obstante, y en apego a los parámetros didácticos de la gamificación, esta tiene como firme propósito, dentro del contexto universitario, premiar el esfuerzo del estudiante que busca con esmero y dedicación el fiel cumplimiento de los indicadores de logros; pero también penaliza la falta de interés sobre el aprender: la gamificación como parámetro cuantificador del aprendizaje en el interior del aula universitaria, ayuda al docente a medir el desempeño de cada estudiante, ya que el educador puede visualizar un panorama en el que supone un mecanismo para fomentar el trabajo en el aula y retomar un control efectivo del desempeño académico de los educandos.

$\mathrm{Al}$ referirnos a la gamificación como un recurso de apoyo docente sobre el cual se orienta un proceso de enseñanza y aprendizaje, es fácil reconocer en esta (la gamificación) una herramienta de construcción didáctica que busca atender las necesidades de aprendizaje de los estudiantes, logrando además fidelizar a los educandos a que encuentren en dicha estrategia metodológica un mecanismo que facilite el aprendizaje y la útil comprensión de los contenidos académicos abordados en la clase. Para que la gamificación pueda entrar al aula, es necesario que tenga una estructuración compuesta por dinámicas centradas en retos, recompensas, logros, etc., lo cual ayuda al docente a transformar clases formativas, tareas aburridas, en atractivos momentos educativos de aprendizaje significativo, en donde además de mejorar sus resultados académicos llevan a cabo una participación activa de la clase.

Desde la misma tónica de análisis, encontramos el aporte de Kapp Karl M. quien argumenta lo siguiente: "Si bien la gamificación tiene poco tiempo de haber ingresado al aula universitaria, también es cierto que esta ya se está aplicando con éxito por mayor número de docentes, quienes han encontrado en la acción de gamificar las clases, exitosos procesos de aprendizaje; la 
gamificación crece y crece en muchas áreas del diario vivir que no son precisamente educativas tales como el marketing, los recursos humanos, o incluso la gestión de relaciones con los clientes, o incluso a la formación de perfiles gerenciales". ${ }^{6}$ Esta exportación de ideas extrapoladas de la interpretación que hace Kapp sobre la gamificación pone en evidencia creciente interés de la comunidad científica en la aplicación de gamificación a otras áreas del conocimiento, por lo que muchas de las experiencias existentes pueden ser exportadas del ejemplo brindado por la docencia y las mecánicas de juego ampliamente aceptadas por la comunidad estudiantil.

\subsection{El roldel estudiante enla didácticagamificadora}

La construcción de nuevos tiempos y espacios en la educación postmodernista, demanda de ciertos estándares, competencias, requerimientos y medidas de acción destinados a cambiar la forma en la que los estudiantes aprenden, por lo que no es posible mejorar la calidad de la educación sin mejorar la calidad académica de los estudiantes, quienes ven en el hecho educativo la posibilidad de avanzar ordenadamente en la consecución de objetivos o estándares académicos. Por lo anterior es urgente hacer un cambio en el paradigma formativo del docente y motivarlo a que integre en su caudal de conocimiento pedagógico, hacia un acercamiento con determinadas competencias tecnológicas que le ayuden a gamificar la clase, a fin de orientar la intervención educativa al logro de capacidades en el aprendizaje y a buscar en forma permanente a que el estudiante adquiera niveles superiores de desempeño.

6 KAPP, Karl M. The Gamification of Learning and Instruction: Gamebased Methods and Strategies for Training and Education, Edt. Pfeiffer, USA 2012.
Sobre la base de un diagnóstico elaborado por el docente es necesario iniciar toda acción educativa, intentando conocer y elaborar el perfil estudiantil que ingresa al aula universitaria; el docente debe tener la habilidad y la pericia de consolidar las diversas competencias de los educandos, logrando identificar la manera cómo el ejercicio educativo puede atrapar al estudiante en una esfera de motivación que lo lleve a ejecutar en forma productiva las habilidades y competencias innatas en su diaria interacción con la tecnología y vincular su formación profesional con las dinámicas que se encuentran detrás de los videojuegos.

Para canalizar las competencias formativas de los estudiantes y vincularlas a la gamificación formativa de sus clases, se presenta a continuación un análisis de las grandes ventajas que esta estrategia metodológica significa.

\section{Cuadro n. ${ }^{\circ} 1$}

Ventajas de la gamificación desde la perspectiva del estudiante

Ventajas de la gamificación desde la perspectiva estudiantil

1. Busca premiar y reconocer el empeño académico que pone el estudiante durante el abordaje de su proceso formativo.

2. El uso de la gamificación en la clase universitaria le ayuda al estudiante a identificar fácilmente sus avances y progresos de su propio aprendizaje.

3. Cuando el estudiante no pone el esmero y el suficiente empeño para mejorar su rendimiento académico, la gamificación le ayuda a mejorar su desempeño mediante el acercamiento de tecnologías y dinámicas integradoras.

4. La gamificación como estrategia metodológica intenta proponerle al estudiante una ruta clara sobre cómo puede mejorar la comprensión de aquellas materias académicas que se le dificultan en mayor medida, debido al cambio de paradigma que supone desarrollar clases gamificadas. 
Conforme a la óptica docente se detalla a continuación un análisis de las ventajas significativa que representa la aplicación de la gamificación como estrategia metodológica.

\section{Cuadro n. ${ }^{\circ} 2$}

Ventajas de la gamificación desde la perspectiva del docente

\section{Ventajas de la gamificación desde la perspectiva del docente}

1. Estimula la implementación del trabajo en equipo y de un aprendizaje colectivo que busca mejorar la dinámica de aprendizaje en el interior del aula.

2. La gamificación dosifica el aprendizaje con gran efectividad y motiva al estudiante a esforzarse más por sus resultados académicos.

3. Cuando el estudiante no pone el esmero y el suficiente empeño por mejorar su rendimiento académico, la gamificación le ayuda a mejorar su desempeño mediante el acercamiento de tecnologías y dinámicas integradoras.

4. La gamificación como estrategia metodológica intenta proponerle al estudiante una ruta clara sobre cómo puede mejorar la comprensión de aquellas materias académicas que se le dificultan en mayor medida, debido al cambio de paradigma que supone desarrollar clases gamificadas.

\section{La gamificación: Paralelismo de enfoques y encuadre de ideas}

La gran mayoría de análisis versados en la problemática educativa, manifiesta una creciente preocupación por lograr cambios sustanciales en los sistemas educativos universitarios; más allá de cualquier reforma curricular implementada en el seno de la educación superior, se debe priorizar en la reforma de las políticas educativas que busquen subsanar las necesidades o vacíos encontrados en los procesos de formación académica universitaria. La gamificación, como un elemento que potencia la mejora del aprendizaje estudiantil, centra su éxito en una característica esencial, la cual parte de la idea de que es el personal docente quien debe recurrir a elementos del juego para armar una figura estratégica que mejore sustancialmente la calidad del servicio educativo que se brinda al estudiante. Ante ello no es difícil encontrar confusión al interpretar el verdadero significado y la implicación de gamificar una clase.

Véase a continuación en la Figura n. ${ }^{\circ}$ 1, una diagramación explicativa que intenta evitar confusiones al tratar de interpretar las ideas resultantes de la gamificación como metodología docente en relación al enfoque lúdico y al enfoque denominado Game Based Learning.

Para una mejor interpretación de las diversas propuestas metodológicas véase el Cuadro n. ${ }^{\circ} 3$.

\section{$¿$ Qué implica gamificar en contextos educativos?}

Sin duda alguna es sumamente importante reconocer la importancia de la actualización de las estrategias metodológicas docentes que permitan consolidar estrategias de enseñanza, aprendizaje y evaluación; debido a la dinámica compleja del hecho educativo hacia el interior de las universidades, los posibles intentos de mejora en el actuar docente, en donde además se produce y se crean dinámicas formativas de considerables variables de entretenimiento pedagógicamente orientado frente al hecho educativo común y tradicional.

La gamificación se presenta al ruedo formativo como una alternativa más que tiene como principal objetivo influir en el comportamiento de los estudiantes en lo referente a lograr que estos obtengan un aprendizaje significativo derivado del disfrute de la realización de la actividad de aprendizaje conformada con elementos del juego, para lo cual es necesario 


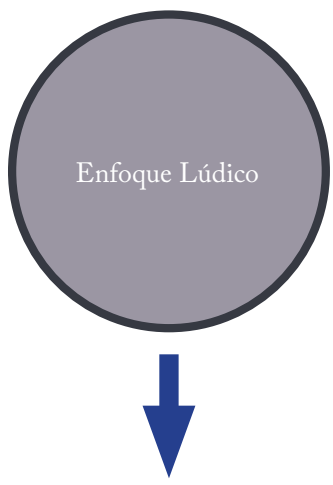

Es el uso de técnicas, elementos y dinámicas propias de los juegos con el fin de potenciar la motivación y reforzar la conducta para solucionar un problema u obtener un objetivo.

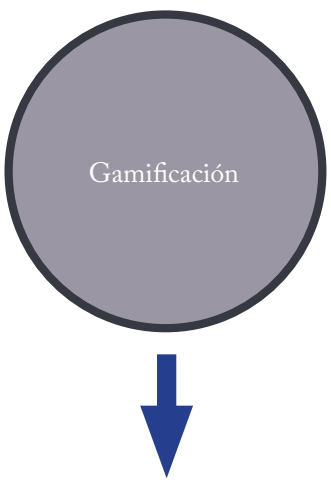

Gamificar es aplicar estrategias (pensamientos y mecánicas) de juegos en contextos no jugables, ajenos a los juegos, con el fin de que las personas adopten ciertos comportamientos

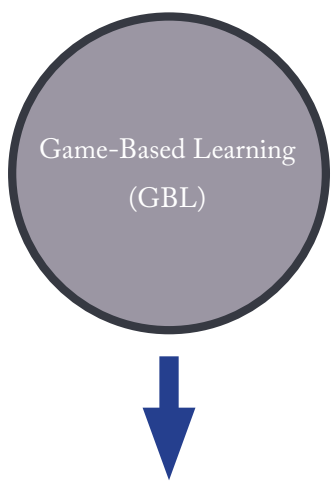

Conjuga el aprendizaje con diferentes recursos conocidos como los juegos, en particular referido a los digitales o de naturaleza computacional, con el fin de apoyar y mejorar la enseñanza.

Figura . $^{\circ}$ 1. Diferencias entre enfoque lúdico, gamificación y el juego basado en el aprendizaje.

\section{Cuadro n. 3}

Disimilitud entre ludificar y gamificar

\section{Diferencias de enfoques e interpretaciones.}

\section{Enfoque lúdico versus Enfoque gamificador}

La palabra lúdico es un adjetivo que califica todo lo que se relaciona con el juego, derivado en su etimología del latín "ludus", cuyo significado es precisamente, juego, como actividad placentera, donde el ser humano se libera de tensiones y de las reglas impuestas por la cultura.

Es el empleo de mecánicas de juego en entornos y aplicaciones no lúdicas, con el fin de potenciar la motivación, la concentración, el esfuerzo, la fidelización y otros valores positivos comunes a todos los juegos.

En resumen: Lo lúdico como aporte a la educación no es nuevo; los antiguos romanos llamaban a las escuelas de primeras letras, "ludus"; y era un "magíster ludi", el maestro que se encargaba de alfabetizarlos, haciéndolos jugar, con letras construidas con marfil o madera. Aprender jugando es una manera placentera, motivadora, y eficiente.

identificar cuáles son los elementos que intervienen en la construcción gamificadora de un proceso de enseñanza y aprendizaje.

Véase a continuación los elementos considerados indispensables para la estructura de una clase gamificada (Ver Figura n. ${ }^{\circ}$ 2).
4. ¿Para qué sirve gamificar una clase desde la perspectiva práctica de la educación superior?

Uno de los principales propósitos de la gamificación del hecho educativo consiste en poder convertir las clases aburridas en acciones de aprendizaje significativo más entretenidas, por 


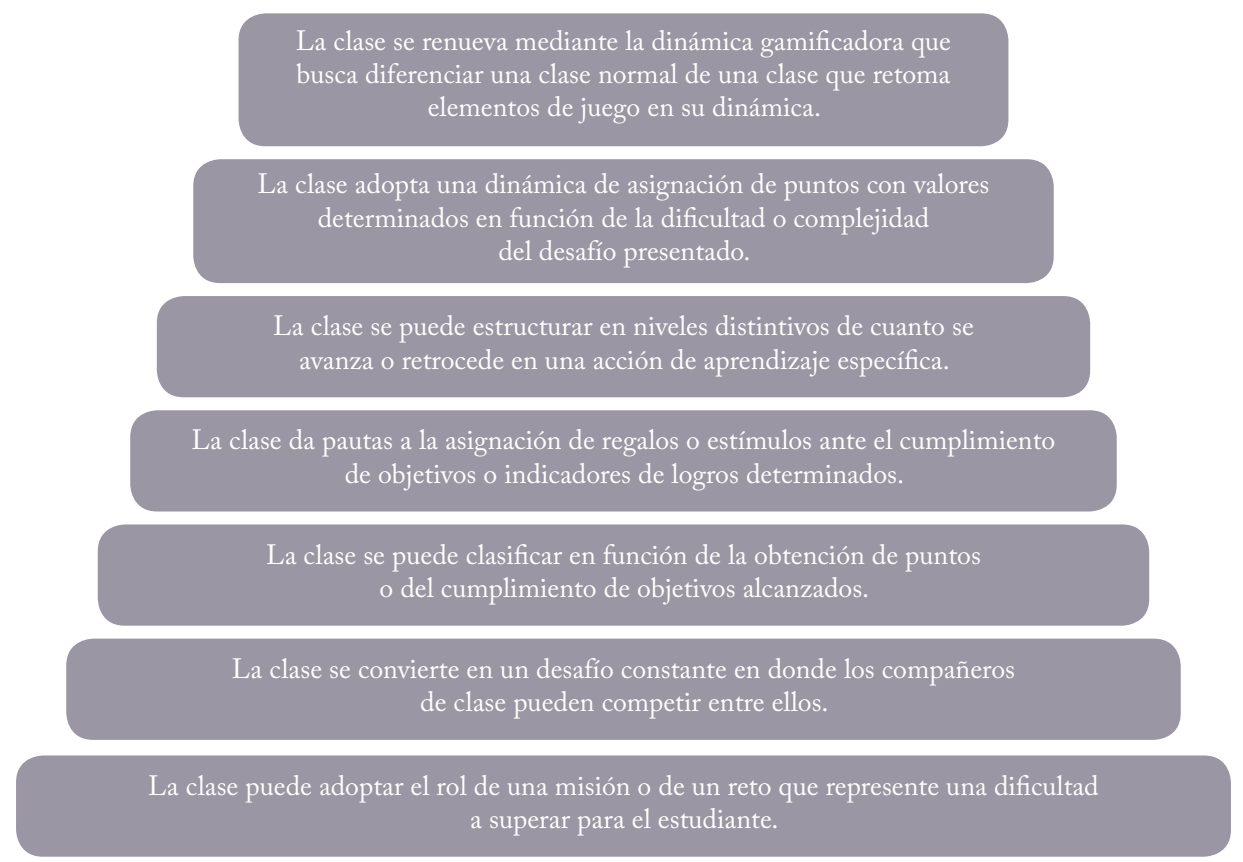

Figura . $^{\circ}$ 2. Elementos considerados indispensables para la estructura de una clase gamificada

lo que la gamificación puede ser utilizada para motivar a nuestros estudiantes a que reinventen el modo y la forma en cómo aprenden. La ejecución de una clase centrada en la dinámica gamificadora se está convirtiendo en la forma por excelencia de fidelización de los estudiantes con alguna materia académica en específico, con lo que se sustituye, mejora, y redinamiza la enseñanza.

Preguntémonos, entonces, ¿cuál es entonces el objetivo académico de la gamificación en el proceso educativo universitario?

Gamificar no es únicamente limitar la clase a la obtención de puntos o recompensas; la gamificación convierte la clase en un hecho divertido en el que se exploran las inquietudes y motivaciones de los estudiantes, lo cual permite conocerlos mejor, ya que un proceso educativo carente de líneas o estrategias gamificadoras, restringe el protagonismo del estudiante, lo cual no contribuye a crear un impulso didáctico que le dé a la clase un espíritu motivacional que pueda fortalecerse con la sorpresa y la recompensa.

Necesariamente el docente debe recordar que un grupo de estudiantes motivados son fácilmente transformados en una clase disciplinada y empeñada en la realización del fiel cumplimiento de los objetivos académicos; por tanto, la condimentación que le da la gamificación al caldo del saber permite el hecho de que desde el estudiante más desinteresado hasta el más atento puedan asumir el reto de aprender y superar sus dificultades de aprendizaje, ya que la gamificación les permite ver el error como algo bueno y superable. 
En resumen, se puede señalar que el objetivo de la gamificación en el proceso de enseñanza y aprendizaje en educación superior, es persuadir al estudiante a que se involucre en el proceso dinámico de transformar una simple clase, en un momento de aprendizaje atractivo y que represente un reto; para lograr esto se deben crear acciones gamificadas que provoquen el deseo de participar e interactuar en la construcción de su propio aprendizaje.

Al respecto Ermi, L., Mäyrä señalaba lo siguiente: "La mecánica gamificadora, hace hincapié en cómo los elementos de una estructura de juegos buscan recompensar a los estudiantes cuando una simple clase adopta el papel de puntajes, emblemas, niveles, tablas de clasificación y los retos. La gamificación por tanto contribuye en la motivación, el comportamiento y la personalidad que ayudan a monitorizar las métricas de aprendizaje establecidas en el currículo, buscando que el docente asegure aplicar principios didácticos y de evaluacion del aprendizaje en el que aprender le implique al estudiante medir un avance en lo que aprende recurriendo a la influencia, la lealtad y la adicción por aprender contenidos de clase implementando gamificación". ${ }^{7}$

A continuación se presenta una Reflexión objetiva al gamificar acciones educativas en la educación superior universitaria (Ver Figura n. ${ }^{\circ}$ ).

5. ¿Para qué gamifico las clases en el nivel universitario?

La utilidad que brinda la estrategia de gamificación aplicada al contexto universitario, permite que una clase pueda superar la conjunción

7 Ermi, L., Mäyrä, F. Player-Centred Game Design: Experiences in Using Scenario Study to Inform Mobile Game Design; 2005.

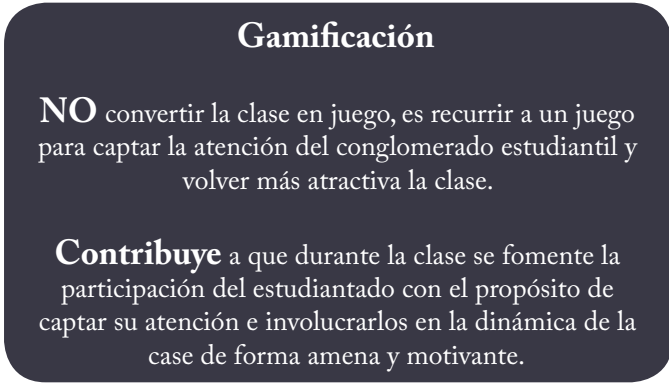

Figura n. $^{\circ}$ 3. Lo que no es gamificación. Elaboración propia.

de conocimientos linealmente teóricos a llegar a consolidar una clase en una amena reunión de aprendizaje, en la que a partir de los elementos que forman parte de la estructura del juego se pueda crear una conectividad que alimente el interés y el compromiso del estudiante por aprender. Cuando el catedrático logra encontrar el exquisito sabor que deja la gamificación en una clase, este fácilmente puede alcanzar mejores niveles de participación por parte de los educandos, de manera dinámica y proactiva, en acciones que generalmente requieren de un esfuerzo de académico vinculado con la eficiencia y la eficacia en el aprendizaje. Es importante que la docencia a nivel de toda América Latina pueda ir cada día integrando más y más las dinámicas de juego en entornos no lúdicos, ya que el crecimiento exponencial del uso de juegos y videojuegos en los últimos años ha despertado el interés de expertos en muchas áreas del campo académico universitario, en donde una Facultad de Medicina, por ejemplo, puede dotar de conocimientos a los futuros galenos por medio de simuladores que ayuden a aprender las técnicas básicas para atender un parto. Como el ejemplo anterior son muchos los ámbitos en los que se puede gamificar por medio 
de una serie de experiencias del estudiante en contacto directo con su aprendizaje, aportando de esa manera un mayor atractivo y motivación a la materia gamificada.

La urgente necesidad que demanda una divulgación masificada es el hecho de transmitir la idea a todo el conglomerado docente; de que la intervención didáctica de una clase a partir de la gamificación, permite construir dinámicas de juego diseñadas en forma puntual para responder a las necesidades e inquietudes de los estudiantes, permitiéndonos así conocer qué es lo que les motiva a aprender con mayor ahínco, empeño y prontitud. Un excelente aporte sobre la clara idea de gamificar una clase la proporciona la obra de (Lee,J.J.\&Hammer,J.2011) en donde se hace una clara alusión al siguiente punto a tratar: "Debido a lo reciente que tiene el concepto de gamificar clases, es difícil hacerle entender al estudiante que el aprendizaje puede construirse sobre la simiente del juego serio, el cual no tiene por finalidad la diversión o el entretenimiento, gamificar supone la creación de condiciones que hagan de la clase un espacio de aprendizaje en tiempo real, que debe funcionar con una gran visibilidad y trazabilidad con la ayuda de los elementos del juego a fin de propiciar experiencias de aprendizaje valiosas que no sean aplicadas constantemente, todo ello con el objetivo de darle siempre al conglomerado académico un sentido de innovación de la clase el cual se genera por la nueva experiencia”.

A continuación se presenta una vinculación entre las mecánicas y las dinámicas de una clase gamificada. (Véase Cuadro n. ${ }^{\circ}$ )

Viendo la figura anterior se puede interpretar que la gamificación como estrategia educativa que gana cada día más adeptos en función de las técnicas y los programas que utiliza de forma complementaria para lograr que la consecución de objetivos educativos sea exitosa al momento de enriquecer una clase mediante un conjunto apropiado de mecánicas de juego que permitan crear una experiencia centrada en la búsqueda de una recompensa que se pueda alcanzar un estatus, que facilite la obtención de logros, que dé espacio para generar una sana competición y que fomente en mejor manera la atención, la concentración y la dedicación por el estudio.

Nunca se debe olvidar que en todo momento la gamificación pretende que el estudiante reflexione y valorice cuál es el contenido metodológico docente que le hace comprender que una propuesta de aprendizaje centrada en el juego puede ser tan efectiva en términos de su concentración, participación, dedicación y triunfo, para aplicar esos mismos principios a otras áreas del conocimiento. Sin embargo, hablar de gamificación no necesariamente es sinónimo de hablar de dispositivos electrónicos y medios digitales, ya que recordemos que una acción educativa estructuralmente gamificada se basa en tres principios básicos que bien pueden aplicarse para un juego de mesa: la mecánica del juego, las dinámicas de juego y los componentes del juego.

Si el estudiante logra identificar y aplicar los tres principios básicos en que se basa cualquier tipo de juego: la mecánica del juego, las dinámicas y los componentes del juego, será fácil para el docente involucrar a los educandos en forma dedicada y divertida en las actividades que se pretenden realizar, ya que será precisamente en esas acciones divertidas en las que podrá encontrar una clase que le conlleve a ganar puntos, subir de nivel, 


\section{Cuadro n. ${ }^{\circ} 4$}

Entre la mecánica y la dinámica gamificadoras

educativas $^{8}$

\begin{tabular}{|c|c|}
\hline $\begin{array}{l}\qquad \text { MECÁNICAS } \\
\text { La aplicación de mecánicas en la gamificación educativa trae } \\
\text { consigo la potenciación de una motivación por aprender en } \\
\text { el estudiante. }\end{array}$ & $\begin{array}{l}\text { DINÁMICA } \\
\text { La aplicación de la dinámica en la gamificación educativa trae } \\
\text { consigo el fomentar en el estudiantado la relación vinculante } \\
\text { entre el conocimiento, el aprendizaje y los resultados. }\end{array}$ \\
\hline $\begin{array}{l}\text { El puntaje: es lo que le asigna el valor cuantitativo a la } \\
\text { gamificación. }\end{array}$ & $\begin{array}{l}\text { Busca una gratificación, retribución o recompensa, con el } \\
\text { ánimo de diferenciar los posicionamientos alcanzados en } \\
\text { forma individual o grupal. }\end{array}$ \\
\hline $\begin{array}{l}\text { Niveles: marcan el avance en el transcurso del camino del } \\
\text { aprendizaje. }\end{array}$ & $\begin{array}{l}\text { Consolidar el posicionamiento adentro de la acción de } \\
\text { aprendizaje. }\end{array}$ \\
\hline $\begin{array}{l}\text { Posesiones virtuales: constituyen la materialización de las } \\
\text { pertenencias que vamos obteniendo. }\end{array}$ & Obtener claridad en cual debe ser el logro a obtener. \\
\hline $\begin{array}{l}\text { Las clasificaciones: son las marcas o pautas que nos indican } \\
\text { el nivel logrado en comparación con los demás estudiantes. }\end{array}$ & $\begin{array}{l}\text { Marcar la pauta competitiva y de posicionamiento entre los } \\
\text { participantes. }\end{array}$ \\
\hline $\begin{array}{l}\text { Los desafíos: estos nos indican los obstáculos que debemos } \\
\text { afrontar durante la acción gamificadora, pero en forma de } \\
\text { competencia. }\end{array}$ & $\begin{array}{l}\text { Acá se estandariza la transformación del propósito elemental } \\
\text { de la acción educativa gamificada. }\end{array}$ \\
\hline $\begin{array}{l}\text { Premios o retribuciones: estos representan el estímulo } \\
\text { ganado al finalizar con éxito las pautas de juego establecidas } \\
\text { (alcanzar los indicadores de logros o cumplir con los } \\
\text { objetivos). }\end{array}$ & $\begin{array}{l}\text { Busca una gratificación, retribución o recompensa, cuando se } \\
\text { cumple fielmente el objetivo de aprendizaje. }\end{array}$ \\
\hline
\end{tabular}

obtener premios, destacar en posiciones de clasificación o triunfar en desafíos y misiones. En opinión de un especialista en gamificación educativa, (BERLUHESHT SELEYGTH, 2009), "la gamificación nos ofrece un refuerzo positivo, instantáneo, sin tener que esperar a una evaluación cuantitativa final, esto facilita una postura estudiantil más receptiva para lograr que su aprendizaje le permita lograr objetivos personalizados por niveles y micro objetivos cuantificables. La aplicación útil del enfoque gamificador en educación es un elemento clave para la disminución del fracaso escolar". (Ver Figura n. ${ }^{\circ}$ )

8 Extraído de ROSS JOHANSON Loui Webster; Towards the gamification of the new educational path; southampton university Education School 2011.

\section{El aula universitaria: Del modelo expositivo al modelo gamificador}

E1 desarrollo transitivo en el que se pasa de una propuesta docente, donde el profesor es el centro del hecho educativo hasta llegar a generalizar la aplicación de clases bajo la propuesta gamificadora, nos lleva al análisis específico sobre cómo podríamos mejorar el aprendizaje de nuestros estudiantes, lo que implica una rigurosa verificación crítica del desempeño del docente al momento de enseñarles y especialmente revisar todo lo que ellos hacen y podrían hacer para motivar a los conglomerados estudiantiles a aprender más contenidos y en mejor forma. Mejorar el aprendizaje de los estudiantes supone poner a prueba la efectividad de las 
metodologías y con ello exigirle al docente el completo abandono de su zona de confort, en donde tendrá que desechar sus prácticas y cómodas metodologías expositivas tradicionales hasta cambiarlas por otras más eficaces, logrando con ello potenciar, introducir y desarrollar los idóneos aprendizajes que tanto se añoran para los estudiantes.

Un primer paso para crear los cambios positivos aplicando clases gamificadas, es consolidando la articulación de aquellos elementos derivados del juego y cómo se podrían incluir en una clase formativa; para lograr tal efecto se necesita que el rediseño de la clase pueda mejorar los resultados de aprendizaje estipulados en los objetivos de cada uno de los contenidos del programa académico. Siguiendo la misma tónica de análisis, también es importante generar una relación vinculante entre la clase gamificada y el proceso de evaluación, es decir, que el estudiante tenga la oportunidad de ser evaluado en cada momento sin percibir que está siendo evaluado, de esa forma se podrá dotar a la clase, de mayor poder formativo y posibilitando una retroalimentación constante que permita fortalecer cognitivamente los mecanismos de retención, reflexión y comprensión del conocimiento.

Queda claro entonces que trasladar una práctica docente universitaria del modelo expositivo al modelo gamificador, contribuye en gran medida a generar reflexiones sobre lo que aprenden los estudiantes, ¿cómo lo aprenden? y ¿para qué lo aprenden? Esto se debe a que la gamificación como recurso de mejora metodológica parte del juego para ayudarle al estudiantado a establecer sus propias motivaciones sobre lo que quiere aprender, teniendo claro que es el mismo educando, precisamente, quien deberá de ocuparse por obtener las recompensas, el reconocimiento o prestigio, los logros y fomentar su propio compañerismo en cada una de las clases en las que participe. Es entonces cuando la gamificación, después de haber potenciado el marco reflexivo de la operación crítica y analítica de las mecánicas de juego, logra solidificar su estructura de aprendizaje para lograr darle el rol protagónico al docente, a fin de que este pueda abrir el camino del aprender en forma congruente y significativa.

A continuación se presenta una valoración objetiva del rol modificador de las acciones y transformaciones que se buscan con la gamificación educativa. (Ver Figura n. ${ }^{\circ}$ ).

\section{7.¿Quélogramos al gamificar el proceso educativo?}

En la actualidad y con la gran cantidad de distractores tecnológicos existentes la tarea del educador se vuelve más difícil, lo cual repercute en el hecho de que nuestros estudiantes no logran dedicarle el tiempo necesario al estudio, por lo que es imposible desarrollar los tan añorados aprendizajes exitosos. En buena parte del hecho educativo, el docente tiene que ponerse como objetivo primordial de su labor educativa el desarrollar competencias formativas en los estudiantes que permitan poner en relieve el cúmulo de conocimientos adquiridos; también es imperante que el docente busque cómo potenciar la capacidad de los educandos para razonar creativa y críticamente, ante lo cual vemos en la gamificación la estrategia idónea para tales propósitos.

Véase a continuación una serie de aspectos positivos que se pueden lograr mediante el uso de la clase gamificada en el contexto universitario. 


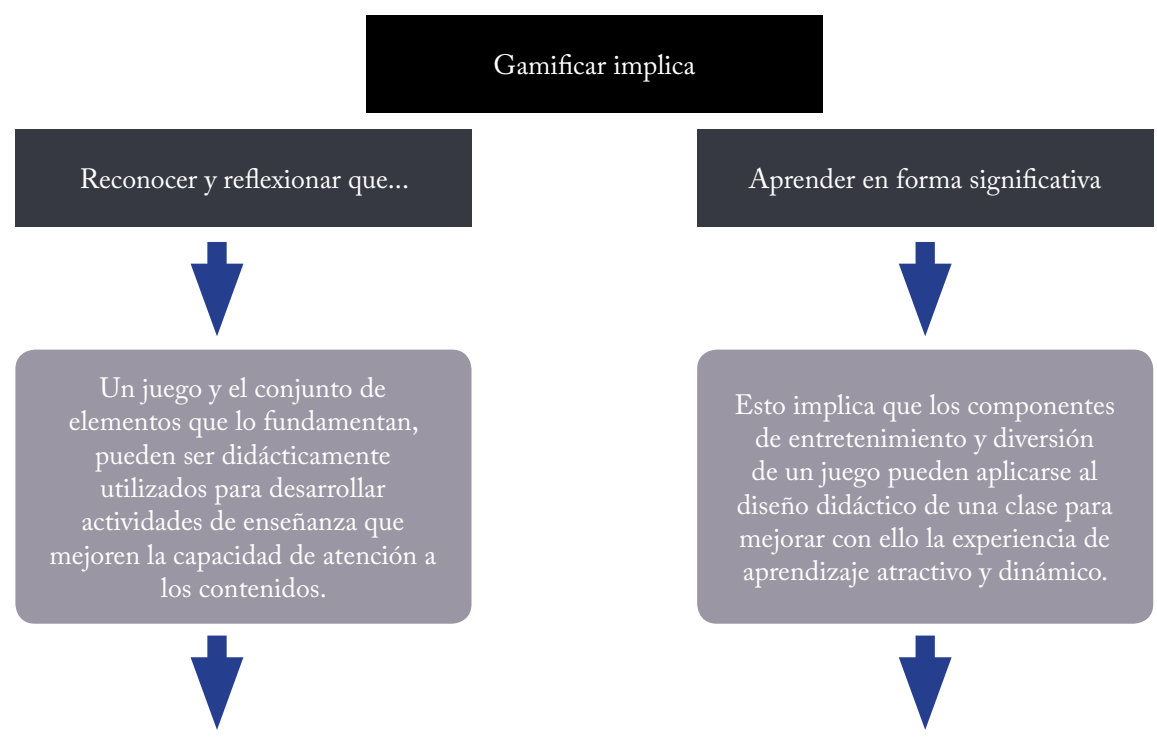

La gamificación debe, por tanto, penetrar en sustancia la nueva forma de hacer clases y lograr cautivar majestuosamente al estudiantado para que el interés por aprender sea cada vez más intenso en su dinámica educativa, logrando que el docente busque explotar el alto valor formativo que tienen los juegos como tecnología auxiliar formativa.

Figura . $^{\circ}$ 4. ¿Qué implica gamificar?

\section{Elementos del videojuego}

Hacer

Sentir

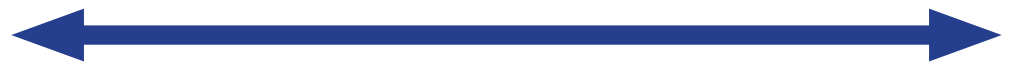

\section{Enfoque en Problemas}

Elementos de Juego (Inductivo)

Cambiar de usuario

Análsis de Datos

\section{Contexto Áulico}

Enfoque en Experiencias Diseño de Juegos (Deductivo)

Impresionar al usuario Observación

Figura n. $^{\circ}$ 5. Esquematización del rol modificador para las acciones educativas gamificadas. Fuente: extraído de: https://ricgooz.files.wordpress.com/2014/05/imagen1.png / fecha de consulta 7 septiembre 2016. 
a) Mediante el uso de la gamificación el docente puede generar con sus estudiantes una comunicación bidireccional muy fluida, creando una dinámica de interacción más cerrada entre estudiante y docente, posibilitando una mejor reflexión de contenidos, usando los elementos del juego para consolidar marcos de retroalimentación académica.

b) El abordaje de contenidos presentados en forma de interrogantes con opciones de respuesta alternativa le dan al estudiante una notificación más rápida sobre la respuesta correcta o la incorrecta, valorando sus estrategias de juego para seguir alcanzando nuevas metas materializadas en conocimientos, y obtener de las respuestas incorrectas experiencias de cambio para mejorar en todo momento.

c) En la gamificación aplicada al aula universitaria se genera una retroalimentación inmediata para incentivar al estudiante a seguir participando y motivarlo a profundizar sus acciones en pos de estrechar lazos con las metas de aprendizaje establecidas. Con la retroalimentación insertada en la clase gamificada se presenta una detección y solución oportuna de los problemas de comprensión y colectivización del conocimiento, en función de las dificultades que manifiestan los estudiantes durante algunas clases en particular.

d) El sistema de gamificación implica a la mayoría de los educandos, estudiar la mayoría de los temas vistos en clase, ya que demanda un estudio previo de los contenidos, con lo cual se crean las condiciones ideales para una interacción presencial de calidad y altamente interactiva, en donde se puede extraer de los errores de aprendizaje de los estudiantes información relevante para que el profesor replantee cómo usar mejores metodologías y estructurar el tiempo de clase para obtener resultados destacados en las evaluaciones del aprendizaje y así evitar el fracaso académico.

\section{Aproximación epistemológica del juego y la gamificación en el proceso de enseñanza y aprendizaje}

El justificar la unificación de elementos del juego con estrategias didácticas que faciliten la obtención de mejores formas de comprender y reflexionar los contenidos por parte de los estudiantes, permite dar paso a la creación de un planteamiento epistemológico en el cual se pueda demostrar la vital importancia de modificar las formas de enseñanza y volver estas más atractivas e interesantes para los estudiantes. Al respecto, el célebre académico especialista en temas educativos Oscar Picardo Joao presenta una concepción epistemológica sobre cómo el juego tiene un rol fundamental en los mecanismos de aprendizaje, lo cual podría interpretarse como un primer acercamiento a los principios didácticos que dieron lugar a la creación de la corriente gamificadora. Sobre el tema Picardo señala que "El significado de aprender y el aprender en forma significativa, es parte del discurso constructivista, en el cual se asume que el estudiante debe ser el protagonista del aprendizaje, en donde se deberán tomar en cuenta e integrar las experiencias previas, al respecto del tema a enseñar, además se debe retomar que el rol del docente se centra en la 
mediación facilitadora de los contenidos, dándole la debida importancia a las posibilidades del entorno como espacio interactivo" (PICARDO JOAO, 2016).

Partiendo de la reflexión que hace Picardo Joao, se pone al ruedo del campo reflexivo la idea de que una estrategia como la gamificación, busca en todo momento que el estudiante sea el protagonista del hecho educativo, llevando a colación el análisis de importantes referentes postmodernistas en el campo de la pedagogía; Picardo argumenta que el autor Cesar Coll, aboga por un nuevo enfoque de aprendizaje comunitario o aprendizaje social, en donde se deduce que la efectividad de un aprendizaje pasa en un primer momento por ser significativamente importante para el estudiante, lo cual pretende que sea el estudiante quien deba descubrir un sentido peculiar de lo que está aprendiendo, y ello sólo se puede lograr cuando el aprendizaje tiene una aplicación real a la vida, o cuando él descubre en el contenido estudiado lo que le es útil o necesario.

Picardo ha señalado que el juego en el ámbito educativo es la manera simbólica en la que se le enseñan las reglas al estudiante, para explicarlo, acude a la postura de Jean Piaget, para demostrar cómo la observación del juego en la formación educativa ha ido evolucionando en concordancia con el desarrollo del conocimiento. Retomando al célebre académico en cuestión, es viable señalar que el juego es un elemento solidario de la inteligencia y se determina por la estructura intelectual en donde prevalecen las inteligencias múltiples de los estudiantes. Por lo tanto, y con base en el criterio gamificador que nos brinda la aproximación epistemológica de la gamificación, es válido señalar que si un docente incide positivamente en el aprendizaje del estudiante, demostrándole por qué es importante aprender, este éxito se deberá en gran medida al rumbo que tome la estrategia del profesor para darle ese toque de vital interés a la clase; de lo contrario, el docente deberá tomar conciencia de que si no logra esa dinámica de eficiencia y eficacia en el aprendizaje, será mejor no intentar gamificar el abordaje de los contenidos.

Paola Scandinava Loreto (LORETO VIVAR, 2015), experta en metodologías del aprendizaje basado en juegos, señalaba lo siguiente: "No es fácil trasladar una clase de la metodología expositiva a sintetizar una clase mediante la gamificación, esto se debe a la imperiosa necesidad de sacar al docente de su zona de confort para exigirle que entregue un poco más de empeño en generar una estrecha relación entre los recursos tecnológicos y las didácticas de la enseñanza”. Desde este argumento y poniendo la mirada crítica más allá de la relación entre gamificación y el aprendizaje basado en el juego, Loreto Vivar intenta manifestar que gamificar contextos educativos implica poner al estudiante en el papel de jugador en la construcción y búsqueda del aprendizaje a partir de las experiencias próximas, pero no exactas a la ludificación.

A continuación se presenta un marco comparativo en el que se acentúan las diferencias entre gamificación y aprendizaje basado en juegos a fin de comprender los propósitos y objetivos que persigue cada una de estas metodologías.

(Ver Figura n. ${ }^{\circ}$ ) 
1 Se centra en utilizar mecánicas de (video) juegos en entornos no lúdicos.

2 Se le pide al participante cumplir con ciertas tareas para obtener recompensa.

3 La motivación es enteramente opcional.

4 Usualmente es fácil y barata de crear/estructurar.

5 Los elementos de un juego se pueden ensamblar.

Figura n. $^{\circ}$ 6. Gamificación versus el aprendizaje basado en juegos ${ }^{9}$

Para comprender el proceso educativo gamificado, se debe recurrir a un análisis psicológico del estudiante para desarrollar de esa forma una planificación de clases centrada en el desarrollo psicocognitivo del conglomerado estudiantil, en donde los educadores acudan al auxilio de una pedagogía del juego, cuya visión educativa sea cimentar senderos viables sobre la forma de desarrollar las clases con un amplio sentido de dinamismo y entretenimiento.

En forma sucinta, lo que se pretende es demostrar cómo la gamificación tiene vínculo directo con otras ciencias para justificar su utilidad en la enseñanza de cualquier campo de la ciencia; pero además permite que el conocimiento científico tenga otros medios de masificación, comprensión y empoderamiento, gracias a esta nueva forma de
1 Usa (video) juegos con fines didácticos.

2 Los juegos tienen un objetivo de aprendizaje especifico.

3 La mayoría de las veces, el sólo jugar resulta gratificante.

4 Es más complicado y costoso de desarrollar.

5 El contenido se adapta a la estructura del juego. utilizar elementos del juego en el campo de la educación formal universitaria.

En resumen, es importante dejar claro ante la comunidad científica pedagógica que la gamificación supera su postura de una simple metodología entretenida y divertida, ya que debido a su dinámica integradora del conocimiento, la gamificación se vuelve un recurso formativo utilizado para acceder a la organización intelectual del individuo, determinando así el funcionamiento cognoscitivo en ciertos mecanismos de cómo aprende el estudiante, transformando la clase en un espacio donde converge la creación de situaciones de aprendizaje, mostrando la actividad constructiva del estudiante.

A continuación se presenta un cuadro relacional en el que se justifica la intervención de la gamificación y el aprendizaje, basado en juegos para la mejora continua del proceso de enseñanza y Aprendizaje. (Ver Figura n. ${ }^{\circ}$ 7)

9 Extraído de http://info.shiftelearning.com/blogshift/gamificacion$\mathrm{y}$-aprendizaje-basado-en-el-juego 
1 Se reemplazan las calificaciones por niveles.

Se busca propiciar o modificar 2 comportamientos, por lo tanto, "perder" no es una opción.

3 Difícilmente se le presenta una elección al participante en el curso.

4 La motivación del participante depende del sistema de recompensas.

5 El aprendizaje se mide por la cantidad de logros y niveles adquiridos.

Figura $\boldsymbol{n} .^{\circ}$ 7. Justificación relacional entre la gamificación y el aprendizaje, basado en juegos para la mejora continua del proceso de enseñanza y aprendizaje universitario. ${ }^{10}$

La relación entre los componentes que forman parte del eje gamificador y del aprendizaje basado en juegos, permite evidenciar como en ambos casos los tópicos educativos relacionados con la metodología, la evaluación y las experiencias de aprendizaje, conforman la nueva dinámica de sustentar la modernización didáctica del aprendizaje. Véase a continuación un diseño lineal del alto impacto que adquiere la gamificación en la formación educativa universitaria (Ver Figura n. ${ }^{\circ}$ 8).

\section{Conclusiones}

Una forma de cerrar este análisis de intervención académica sobre como la gamificación puede ser una estrategia metodológica que enriquece la

10 Extraído de http://info.shiftelearning.com/blogshift/ gamificacion-y-aprendizaje-basado-en-el-juego Promueve el pensamiento critico y la
resolución de problemas.

2 Es posible "perder" en el juego ya que hay reglas y objetivos a cumplir.

3 Puede incluir simulaciones para que el participante aprenda de sus experiencias.

4 Los participantes se divierten al completar los objetivos del juego.

5 Los participantes se divierten al completar los objetivos del juego.

dinámica educativa universitaria, es retomar la idea de Marcelo Alberdi Alarcon Bonet, quien señala lo siguiente: "El patrón modificador de la nueva didáctica universitaria, parte del aprendizaje basado en problemas y termina en la gamificación como una función de Positive Feedback (retroalimentación positiva) para orientar y reforzar ciertos comportamientos dentro del mecanismo de aprendizaje de cada uno de los estudiantes y lograr que la retención del conocimiento se dé en forma indefinida cuando se logra estabilizar una conducta generalizada que fomente el comportamiento pedagógico deseado". ${ }^{11}$ Por lo tanto, las siguientes conclusiones pretenden crear un panorama educativo en el cual la gamificación pueda expandirse y consolidarse como una excelente herramienta motivadora que reta a educandos y educadores a alcanzar los objetivos de aprendizaje por medio de los juegos, fomentando el incremento por la sana competencia y la

11 ALARCON BONET, Marcelo Alberdi; “Juegos y Aprendizajes. Nuevos panoramas escolares”; Ediciones Cromares, BERTILUGLA Argentina, 2014. 


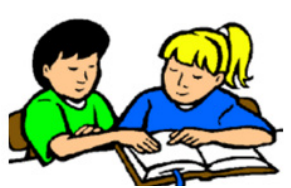

Clases gamificadas generan estudiantes motivados por aprender.

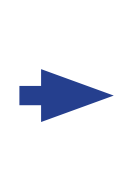

La gamificación motiva y mejora la dinámica grupal de aprendizaje.
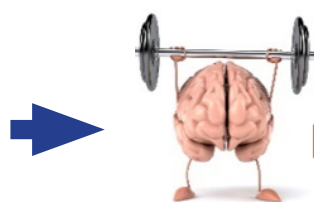

La dinámica gamificadora mejora la retentiva, la capacidad de atención y de crítica reflexiva del aprendizaje.

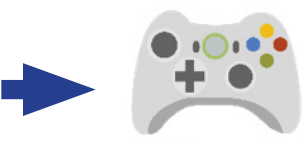

La adopción de elementos del juego en la dinámica educativa, favorece las habilidades y actitudes positivas del aprendizaje significativo.

Figura n. $^{\circ}$ 8. Diseño lineal de la gamificación en la formación educativa universitaria.

Fuente: elaboración propia.

incorporación de reglas, resultados cuantificables y parámetros entretenidos que le dan el atractivo realista al hecho educativo.

\section{Por lo tanto, se concluye:}

1. Que una clase gamificada se sostiene con argumentos centrados en el juego pero implementados como experiencias de aprendizaje que utilizan los retos en situaciones y elecciones reales cuyo nivel de dificultad vaya en aumento, por lo cual una clase gamificada deberá contar con una historia atractiva que permita convertir los objetivos de aprendizaje en retos por superar.

2. Que la introducción de la gamificación es importante debido a que adquiere el rol de herramienta motivadora, ya que esta puede transformar la motivación extrínseca en intrínseca, logrando con ello despertar la pasión del estudiante por el aprendizaje y una enorme necesidad por aprender significativamente, valorando que el desarrollo de una actividad gamificadora es en beneficio del mismo estudiante.
3. Que el éxito de una clase gamificada en el contexto superior universitario deberá tener una serie de objetivos diarios de conformidad a su planificación metodológica, la cual habrá de contar con un sistema para medir el progreso de los mismos; esto se debe a que un sistema educativo que recurra a la gamificación tiene garantizadas la reducción del estrés y los niveles de ansiedad, poniendo mayor atención al involucramiento en el juego.

4. Que la gamificación como soporte de la estrategia docente universitaria elimina la incertidumbre sobre ¿para qué aprendo? y ¿con qué aprendo?, así como la monotonía del modelo expositivo, ya que la puesta en marcha de una actividad gamificadora le ayuda tanto al estudiante como al docente a conocer de manera objetiva el nivel de progreso en el aprendizaje personal de cada individuo sobre la base de sus propios intereses. 


\section{BIBLIOGRAFÍA}

Berluhesht Seleygth,J.M. (2009).Teknik Trender i utbildning. Uppsala Sweden: Uppsala Universitet Upplagor.

Lee, J.J. (2011). Gamification in Education: What, How, Why Bother? Academic Exchange Quarterly.
Loreto Vivar, P. S. (2015). Fundamento del juego serio en contextos educativos. Lima, Peru: Universidad Federico Villareal EDICIONES.

Picardo Joao, O. (2016). Teoria curricular. San Salvador: UFG Editores. 